\title{
EDUCACIÓN A DISTANCIA: TRANSFORMACIÓN DE LOS APRENDIZAJES
}

\author{
Liliana Yonué Covarrubias Hernández* \\ https://orcid.org/0000-0002-8111-6579
}

RECIBIDO: Octubre 2020 / ACEPTADO: Noviembre 2020 / PUBLICADO: Enero 2021

\begin{abstract}
Como citar: Covarrubias Hernández, Liliana. (2021). Educación a distancia: transformación de los aprendizajes. Telos: revista de Estudios Interdisciplinarios en Ciencias Sociales, 23 (1), Venezuela. (Pp. 150-160).

DOI: www.doi.org/10.36390/telos231.12
\end{abstract}

\section{RESUMEN}

El objetivo de este artículo es reflexionar en torno al nuevo contexto educativo que la comunidad estudiantil y docente enfrentan luego de atravesar por la situación pandémica a la que la Organización Mundial de la Salud nos remitiera con la declaración del Covid-19 como nueva enfermedad en contagio a nivel mundial, dando lugar así a modalidades virtuales como la nueva normalidad en que se imparten los aprendizajes a la par del temor y la desigualdad social que impera de manera generalizada. Apoyados en la reflexión y análisis teórico de algunas investigaciones y perspectivas teóricas como las trazadas por Díaz (2020), Zebadúa (2020), Mancera, et.al. (2020), Silva (2020), Truco (2020), Trejo (2020) y en los recientes estudios de la CEPAL (2019), observamos los nuevos comportamientos en torno a la continuidad de los procesos educativos que se dieron principalmente en América Latina donde la alfabetización digital sigue siendo un pendiente de los gobiernos. Por tanto, se hace necesario observar como propuesta la humanización de los agentes educadores, del sistema y la sociedad en general para contribuir con esta nueva modalidad de acercar los conocimientos a los educandos de los diferentes estratos sociales, generar empatía para comprender que la gran mayoría no cuenta con acceso a las herramientas tecnológicas y mucho menos a la manipulación de éstas, de tal forma que se requiere la injerencia de todos los sectores para seguir construyendo y formando a las nuevas generaciones.

Palabras Clave: Aulas virtuales; Educación a distancia; Transformación educativa; Individualización de los aprendizajes; Nuevos agentes educadores.

\section{Distance education: transformation of learning}

\section{ABSTRACT}

The objective of this article is to reflect on the new educational context that the student and teacher community face after going through the pandemic situation to which the World Health

\footnotetext{
* Profesor investigador en la Universidad Pedagógica Nacional (UPN) Unidad 061 y la Universidad del Valle de Atemajac (UNIVA) Campus Colima. Integrante de la Red de Investigadores Latinoamericanos Unidos por la Democracia y la Paz, www.insumisos.com. Correo electrónico: lomismo255@yahoo.com.mx
} 
Organization referred us with the declaration of Covid-19 as a new contagious disease worldwide, thus giving rise to virtual modalities such as the new normal in which learning is taught along with fear and social inequality that prevails in a generalized way. Supported by reflection and theoretical analysis of some research and theoretical perspectives such as those outlined by Díaz (2020), Zebadúa (2020), Mancera, et.al. (2020), Silva (2020), Truco (2020), Trejo (2020) and in the recent studies of the CEPAL (2019 and 2020), we observe the new behaviors around the continuity of the educational processes that took place mainly in Latin America where digital literacy continues to be a pending issue for governments. Therefore, it is necessary to observe as a proposal the humanization of educational agents, the system and society in general to contribute with this new modality of bringing knowledge closer to students from different social strata, generating empathy to understand that the vast majority It does not have access to technological tools and much less to manipulate them, in such a way that the interference of all sectors is required to continue building and training new generations.

Key words: Virtual classrooms; Long distance education; Educational transformation; Individualization of learning; New educational agents.

\section{Introducción}

Tradicionalmente se reconoce a la radio, la televisión, los video y audio casetes, como aquellos artefactos que dan entrada a las nuevas tecnologías de la información (Technologies of information and communication TIC, por sus siglas en inglés), las que se insertan como novedosas formas de comunicar e informar, y asimismo aportar en la innovación de los métodos para acercar información en el proceso de enseñanza-aprendizaje. No obstante, luego de los años 70's y 80's el interés por modernizar la comunicación, la gestoría de información y los nuevos conocimientos, fue ampliando el abanico de variantes que dieron pauta a la existencia de tecnologías innovadoras que poco a poco se fueron posicionando no solo en el interés de la sociedad del conocimiento, sino como diversas formas de entretenimiento, de aprendizaje y métodos de apoyo en el ámbito educativo.

Más en esa búsqueda por posicionar a la tecnología como parte fundamental de los procesos educativos, terminó por ubicarse tan solo como una simple aplicación lúdica, a la que podía acudirse de vez en cuando si se deseaba, esto es, que fue necesario imprimir el razonamiento, la lógica e inteligencia humana para que las tecnologías tuviesen ese impacto educativo que desde hace décadas se ha buscado impulsar (Viteri, 2011).

Sociedad del conocimiento, sociedad digital o sociedad de la información, lo cierto es que con la introducción generalizada de las nuevas tecnologías de la Información y la comunicación a todos los ámbitos de nuestras vidas se produjo un cambio significativo en nuestra manera de trabajar, de relacionarnos y de aprender; estableciendo así nuevas formas de integración de los usuarios con los dispositivos enlazados a la red y los novedosos mecanismos de su momento, donde se modificaron los clásicos roles de receptor y transmisor de información y el conocimiento. De tal manera que esas nuevas formas de circulación de contenidos y aprendizajes sin barreras espacio-temporales trajo además el surgimiento de nuevos conceptos de mediación educativa y que sin intención alguna trastocaron el modelo clásico y/o tradicional de relación entre el individuo, la cultura y la enseñanza. 
Sin duda, la injerencia de las TICs en los procesos educativos vino a dejar especial relevancia, pues en cada rincón del mundo se pretendía la mejora de la calidad del proceso enseñanza-aprendizaje, de tal forma que su aplicación en el campo pedagógico buscaba optimizar los resultados de todo un sistema escolar y al mismo tiempo, llevar el conocimiento a las poblaciones particularmente excluidas.

De esta forma, con base a evidencia empírica y en una revisión bibliográfica con selección de material de reciente análisis acorde a la temática consultado en la web, se aborda una breve cavilación teórica de estudios realizados por la CEPAL (2019), y de algunas investigaciones delineadas por Díaz (2020), Zebadúa (2020), Mancera, et.al. (2020), Silva (2020), Truco (2020), y Trejo (2020), así se pretende plasmar en este artículo diferentes reflexiones sobre la situación educativa de hoy en día, y las nuevas modalidades que se extienden trastocando la vida en sus diferentes ámbitos, observando en primer lugar cómo se vive la educación a distancia o educación no presencial, y la manera en que ésta oferta las diferentes formas de aprendizaje con el soporte de las herramientas tecnológicas y el uso de las plataformas virtuales.

Así también se presenta un panorama del desarrollo de la educación en confinamiento aunado a la pandemia Covid-19, misma que evidenció la desigualdad social y económica, y con ello la brecha digital. Además se muestra en los análisis aquí vertidos cómo las aulas virtuales forjaron nuevos agentes educadores que intervinieron en el proceso de enseñanza - aprendizaje ante las medidas del distanciamiento social y las nuevas manera de transmitir saberes, lo que viene a transformar por ende la práctica educativa y en consecuencia a mostrar los nuevos retos de la nueva educación; y finalmente se arriban las conclusiones.

\section{Educación a Distancia}

Abordar la Educación a Distancia es evocar a la nueva modalidad de compartir conocimientos sin el acercamiento físico entre docentes y educandos, más si con la utilización de medios técnicos que permitan la interacción entre éstos, o como bien señaló Martínez (2008) se trata de una estrategia educativa en la cual confluyen los factores de espacio y tiempo, donde incluso la ocupación 0 el nivel de los participantes no condicionen el proceso enseñanzaaprendizaje.

La Educación a Distancia o Educación no presencial surge pues como una alternativa de las sociedades modernas frente al interés de expandir la educación y elevar los niveles de instrucción de grandes grupos humanos, destacando de manera especial a quienes no pueden asistir de manera permanente a los centros educativos, y en el contexto actual por razones de la pandemia. Es así que encontramos términos utilizados para referenciar esta modalidad: Aprendizaje a distancia, abierto, distribuido, entre otros, donde la variedad de medios electrónicos sirven de apoyo para las sesiones educativas en las que docentes y estudiantes habrán de compartir voz, imagen y hacer contacto visual.

Pasando por el manejo de las computadoras, los equipos multimedia, la llegada del internet, las nuevas tecnologías y su incorporación al ámbito educativo promovieron la creación de nuevos entornos didácticos que trastocaron de manera directa a los actores del proceso de enseñanza-aprendizaje y al escenario mismo donde éste se desarrollaba, para dar paso después al e-learning, como primera modalidad donde el proceso de enseñanza-aprendizaje se realizó a través de redes de ordenadores para atender la formación de individuos dispersos 
geográficamente, pero que interactuaban con el docente en tiempos diferidos, dando vida así a las aulas virtuales (Area y Adell, 2009).

Conforme la modernización de las tecnologías de la información y la comunicación se fueron afianzando, se manifestaron otras formas de avances tecnológicos, ligado al desarrollo de las telecomunicaciones, como el m-learning o aprendizaje electrónico móvil, cuya metodología de enseñanza y aprendizaje es apoyarse en pequeños dispositivos móviles: tabletas, iPod, PDA, teléfonos inteligentes móviles, y otros más que tuvieran conectividad inalámbrica (Rodríguez y Coba, 2017).

De esta manera y ante la creciente demanda de educación empezaron a surgir numerosas y diversas ofertas educativas, en su mayoría apoyadas en modalidades diferentes al concepto tradicional como lo fue y ha sido el escolarizado o presencial, dando lugar así a otras formas del proceso educativo que apoyaban la ampliación de la cobertura educativa, pues estas nuevas formas de aprender tenían la ventaja de ofrecer a los estudiantes la flexibilidad de los horarios de estudio para tomar los contenidos, sin que la edad fuera una limitante para seguir aprendiendo en un "centro" educativo. Tales opciones no convencionales se reconocieron por ser "abiertas", "no escolarizadas", "no presenciales", "a distancia", "en línea o virtual"; a las que se sumaron las denominaciones "semiescolarizada", "semipresencial" y "multimodal", que no eran más que una modalidad mixta, donde la clase presencial se combinaba con las sesiones virtuales para reforzar los conocimientos programados y de esta manera apoyarse en plataformas virtuales, con la flexibilidad que las nuevas ofertas educativas ofrecían, apoyadas en la mercadotecnia de los anglicismos online, e-learning, m-learning, entre otras (Torres y López, 2015).

Las instituciones educativas han buscado esa modernización de los contenidos con la inserción de las TIC's que desde su implementación han venido transformando la calidad educativa, por lo que la constante de las universidades públicas y privadas es y seguirá siendo modernizar los procesos a fin de alcanzar también la internacionalización de sus programas, misma que ha sido complicado atender tan solo con la programación en línea, sin los intercambios estudiantiles que brindan a los estudiantes la oportunidad de conocer otras culturas, otros profesionistas, otros proyectos, otros idiomas y nuevos contenidos académicos a partir del cierre de fronteras.

\section{Pandemia Covid-19 y Educación en confinamiento}

Para marzo del 2020, en que el Covid-19 se hace presente a nivel global luego de que la Organización Mundial de la Salud (OMS) lo declarase como enfermedad y en consecuencia como pandemia que trastocó todas las esferas y estratos sociales, el temor social generalizado se palpó en cada rincón, generando así la preocupación por la salud pública, obligando a los Estados a actuar en consecuencia con el cierre no solo de fronteras, sino también de los centros de trabajo, las empresas, las oficinas gubernamentales, y por supuesto las escuelas, donde las medidas de prevención se acentuaron más, pues los docentes y estudiantes fueron los primeros en remitirse al encierro voluntario, transformando así, y de manera drástica todas las dimensiones de la vida particular y social (Zebadúa, 2020).

Las afectaciones que el Covid-19 empezó a dejar a su paso en la población a nivel mundial aunado a las enfermedades preexistentes, generaron que la mayoría de los países recurrieran al distanciamiento social y al confinamiento en sus propias casas, en una cuarentena 
"voluntaria" que hasta hoy se mantiene en varias naciones, pues con las medidas gubernamentales en el afán de preservar la salud pública, también se recurrió al uso de la fuerza para garantizar el control y protección a la misma población (Silva, 2020).

En la búsqueda por mantener ese control, se dispersó por todo el mundo un discurso de pánico y angustia, pues en un principio los organismos e instituciones de epidemiología desconocían las características y las condiciones en que el coronavirus podía propagarse y extender así los contagios masivos. Tanto así, que en el ámbito educativo la emergencia en sus inicios dio lugar al cierre de las instituciones educativas públicas y privadas en más de 190 países, para evitar así la trasmisión del virus, registrando las Naciones Unidas para la Educación, la Ciencia y la Cultura (UNESCO) que más de 1,200 millones de estudiantes en sus diferentes niveles educativos habían "abandonado" las aulas en espera de una modalidad que en específico sustituyera sus clases presenciales (Informe Covid-19 Cepal-Unesco, 2020).

Sin embargo, lo más complicado de esta situación no era la espera de una nueva modalidad virtual que resolviera el problema de la continuidad del proceso enseñanzaaprendizaje, sino la enorme desigualdad social y económica que priva en la mayoría de los países principalmente en América Latina y el Caribe, dejando al descubierto la incesante brecha digital, que terminó por evidenciar que el aprendizaje a distancia no era la solución para todos, pues no todos contaban con el acceso a internet, a dispositivos y tecnologías inteligentes que les permitiese seguir a los docentes y sus conocimientos en línea, y más aún, ni siquiera contaban con un espacio para continuar aprendiendo y estudiar.

Por tanto, resultó un tanto ilógico hablar de la continuidad de los procesos educativos cuando en la mayoría de los hogares no se contaba ni se cuenta con energía eléctrica, ni espacios dignos para ubicarse en el interior de los casas - habitación, cuando no hay alimento para los niños que recibían desayunos escolares, cuando la falta de oportunidades laborales agravadas por la pandemia- no permitía el acceso o la compra de equipos de cómputo y/o dispositivos inteligentes.

Abordar la existencia de la educación a distancia de manera generalizada, sería aseverar que la oportunidad del uso de las plataformas digitales y la conectividad a internet es para todas las comunidades estudiantiles y que la continuidad de los aprendizajes se atendió por parte de los Estados, resolviendo el problema de cobertura digital y las desigualdades económicas que golpean a los más excluidos.

De acuerdo con la CEPAL (2019) para el año 2016 se registraba que aproximadamente el $42 \%$ de la población que vivía en áreas urbanas en América Latina, contaba con acceso a internet en el hogar, mientras que en las áreas rurales tan solo el $14 \%$ tenían tal conexidad, cifras que según la Comisión Económica para América Latina y el Caribe pudieron repuntar en caso de que el acceso al internet móvil fuera creciendo, lo cual fue poco notable, dada la disparidad de oportunidades (Cepal, 2019).

Aunado a ello confinarse en los hogares tanto estudiantes como docentes, obligó a responder a demandas emergentes, pues se hizo necesario replantear las planeaciones de contenidos teóricos y prácticos, esto es, ajustar la metodología de los procesos educativos, rediseñar materiales, currículas, formatos y medios de trabajo en el quehacer docente; asimismo fue preciso atender otras instancias para evitar el "abandono escolar": la salud mental de los estudiantes. Así, la mayor implementación de las nuevas herramientas de trabajo y la capacitación para el manejo de las TIC's en la enseñanza de la educación en sus diferentes 
niveles, junto con el tratamiento socio-emocional que debía dar a los alumnos y padres de familia, se volvió una constante en las dinámicas pedagógicas de la formación docente en los tiempos de pandemia.

De igual forma, otro de los elementos que fueron determinantes en las labores pedagógicas, fue el factor tiempo, pues la necesidad de ajustar las condiciones al ámbito de la educación a distancia, vino a incrementar las responsabilidades docentes, aumentando significativamente las exigencias y los tiempos de trabajo no solo para impartir sesiones y/o conocimientos, sino también para preparar material a compartir virtualmente, revisar las conexiones, dar seguimiento a cada estudiante y sus condiciones propias para continuar en el proceso, a la par de tener que compatibilizar los tiempos del trabajo doméstico, tomando en cuenta que la mayoría de los docentes son mujeres (Informe Covid-19 Cepal-Unesco, 2020).

\section{Aulas Virtuales y nuevos agentes educadores}

La educación a distancia tiene actualmente una relevancia que quizás nunca antes había tenido, la injerencia de las tecnologías de la información y la comunicación (TIC's) se han posicionado con mayor ventaja que los medios tradicionales: la televisión y la radio, cuando eran los canales idóneos para el funcionamiento de las telesecundarias en los años 70's, pues las TIC's permiten acceso a contenidos prácticamente ilimitados, la apertura y vinculación a conversaciones virtuales entre múltiples personas, herramientas diversas y el procesamiento de datos, con lo cual determinan la calidad y cantidad de información que se acerca a los estudiantes (Mancera, et, al., 2020).

De esta forma, hoy se observan nuevos espacios con la libertad para organizar el tiempo de estudio, teniendo como aporte el uso del internet que servirá de base para el proceso de enseñanza-aprendizaje, generando la reducción de costos en algunos aspectos que se involucran, personalizando el aprendizaje de quienes usan tal modalidad, con la ventaja de tener acceso permanente al material didáctico que las plataformas virtuales soportan para ventaja de los usuarios. Es necesario puntualizar que cuando se empezó a abordar el término e-learning como espacios virtuales, se pretendía enfocarlo más como un concepto de mercadotecnia que académico, aunque conforme se avanzó en la modernización de los procesos educativos, terminó por vincularse a la gestión del conocimiento (Area y Adell, 2009).

De tal forma, que las aulas virtuales de hoy en día son los nuevos "campos" de aprendizaje donde el alumno construye buscando responder a las diferentes representaciones formativas que la presencialidad le otorgaba, como el enriquecimiento educativo del servicio social y la práctica profesional en el caso del nivel medio superior y superior, en donde sin duda los actores han tenido la necesidad imperante de establecer alternativas para cumplir tales asignaturas, comprendiendo por supuesto que los resultados no serán los mismos, siendo esa aceptación lo que conducirá a aplicar estrategias por parte de los sistemas educativos, con los nuevos modelos digitales que dan vida a la educación a distancia y sus nuevos enfoques didácticos.

Es de reconocer que la comunidad docente enfrentó el desafío de continuar los procesos de enseñanza-aprendizaje, ajustando currículos y recursos pedagógicos para reforzar los requerimientos de todos y cada uno de los estudiantes, más el reto mayor fue y sigue siendo el atender a la población migrante y a las comunidades indígenas que históricamente padecen 
una profunda desigualdad de la cobertura curricular, evidenciando el estado de rezago mayor al que esta población se relega.

Si la continuidad de los procesos educativos tanto para estudiantes como para los docentes se dio en los hogares y en confinamiento, la situación pandémica evidenció por tanto que en la mayoría de los casos las habilidades digitales se venían registrando como meramente un apoyo en dichos procesos, de manera aislada y con una carencia de destrezas para hacerlo parte fundamental del desarrollo pedagógico, pues desde los años 90's se implementaron tan solo para mejorar los resultados del aprendizaje (Truco y Palma, 2020), pues desde la llegada de las computadoras, el internet y los dispositivos digitales se hablaba ya de la necesidad de usar la tecnología como parte de los procesos educativos de manera cotidiana, de incorporar las TIC's desde la formación básica, más sin éxito alguno, aun cuando la misma OCDE insistía a los distintos gobiernos sobre la importancia de invertir en infraestructura que le asegurase a los docentes estar a la vanguardia en cuanto al diseño y aplicación de herramientas de la educación del futuro (Trejo, 2020).

Por tanto, antes de imaginar que una situación pandémica nos obligaría a tomar la educación a distancia, ya se recibían las recomendaciones de hacer uso de las diferentes tecnologías para responder a los procesos con materiales virtuales, atender la formación pedagógica con la inserción de competencias digitales, ajustando planes y programas de estudio donde la tecnología fuera el soporte principal de los contenidos vistos de manera tradicional.

Ahora bien, si la contingencia sanitaria por Covid-19 obligó a principios del año a que todos los actores implicados en los procesos educativos se sumaran a tales tareas educadoras, uno de los principales agentes que asumió la mayor parte de dicha responsabilidad, fueron los padres de familia, quienes en el interés y preocupación por dar continuidad a la formación educativa de sus menores hijos, asumieron el control de la educación inicial y básica desde sus casas, con sus propios dispositivos y móviles cuando en los hogares no se contaba con equipos de cómputo, - en el mejor de los casos-, pues la desigualdad histórica mostrada en los índices de analfabetismo se hicieron presentes al evidenciar que no todos los padres de familia estaban en condiciones cognitivas para aportar en los aprendizajes formativos de los menores. Así, la familia como "nuevo agente" educador enfrentó también uno de los más grandes retos para seguir brindando a los niños los conocimientos, mismos que evidentemente no fueron recibidos y canalizados con la misma fluidez que el educador se los brinda en el aula, como campo de interacción social con sus pares, donde fluyen las ideas y las prácticas para reforzar los contenidos aprendidos.

Cierto es que las instituciones formativas como responsables principales de la formación educativa tienen la mayor influencia para que estos aprendizajes lleguen a transformar el constructo mental de los estudiantes, sin embargo como parte actora de la sociedad los padres de familia retroalimentan los saberes y la educación se vuelve integral con las prácticas de reforzamiento por parte de éstos últimos en el hogar, más cuando los agentes principales no son profesionistas ni especialistas en la transmisión de saberes y de los procesos cognitivos, los contenidos que los menores estudiantes reciben no pueden fluir significativamente, con mejores resultados y con la misma calidad que le brinda el educador y/o docente en el aula.

\section{Transformación de la práctica educativa: Reto de la Nueva Educación}


Aun cuando desde hace décadas se ha buscado mejorar la manipulación de los objetos tecnológicos para ser incrustados en los procesos educativos, lo cierto es, que las tecnologías por sí solas no constituyen el éxito de la educación contemporánea, no es el único aporte en el desarrollo de las nuevas generaciones. Las nuevas tecnologías de la información y la comunicación aportan en la expansión de la enseñanza y el aprendizaje desde su implementación, más aun en los tiempos de confinamiento por la situación pandémica, que obliga al sistema y a la comunidad implicada a buscar el acercamiento de los aprendizajes por métodos a distancia, aun cuando se siga trabajando en mejorar este desarrollo de competencias que lleva a la búsqueda de la calidad de los procesos y su manejo de información.

Es menester por tanto, promover desde las áreas directivas de la educación, el aprender nuevas funciones evaluativas que le permitan al docente reconocer los avances individualizados de los estudiantes en el marco de las asignaturas y/o programas a cursar, pues es evidente que uno de los vicios con que nace e inicia esta etapa reciente de inclusión de la tecnología en la adquisición de saberes es la de "cumplir", "hacer" y "entregar" por un resultado numérico, más que por un aprendizaje significativo, errores que nacen al no tener identificado plenamente la autogestión del conocimiento por parte de los educandos y la autoalfabetización digital por parte de los docentes como aporte en su labor de profesionalización (Díaz, 2020).

"Cumplir" con la entrega de tareas y actividades se ha vuelto una constante en los estudiantes de los diferentes niveles educativos, dado que la nueva modalidad virtual y el ajuste de los tiempos frente a las pantallas y/o de las videoconferencias remiten a los mismos a "entregar" sin reflexionar, sin entrar en el debate de las ideas, en esas prácticas que en el aula enriquecían los saberes, retroalimentaban los aprendizajes y consolidaban el constructo mental generando estudiantes críticos y con la madurez intelectual para producir y reproducir teorías, pues al individualizar los "saberes" la autogestión de los mismos queda reducida a un mero rezago ante el clima de desapego e incertidumbre que hoy enfrentan.

De igual forma, la alfabetización digital y/o dominio del manejo de las nuevas herramientas pedagógicas basadas en las tecnologías puede ser una preocupación de los sistemas educativos, de los agentes directivos y hasta del Estado mismo; no obstante para poder existir éste debe ser un compromiso contante y directo de la comunidad docente, la que con vocación de servicio al sector estudiantil y a la sociedad misma, habrá de responder buscando acercarse a esos aprendizajes autónomos y permanentes que le permitirá atender la transmisión de conocimientos en un contexto como el que hoy se vive, y del cual no se tiene certeza de superar en un tiempo determinado (Díaz, 2020).

Si bien es cierto que la inserción de la tecnología tiene ya varias décadas en la vida de los individuos, creando nuevas generaciones implicadas en lo tecnológico, con una forma de vida cómoda por la seguridad que la rapidez del internet le deja en los procesos didácticos y de entretenimiento que el mercado digital les deja (Zebadúa,2020), también es un hecho que las formas de aprendizaje en los diferentes niveles educativos formales e informales siguen evolucionando a grandes pasos y habrá que ajustarlos a manera de configurarlos como significativos, pues es evidente que luego de atravesar la educación a distancia obligados por el temor de la pandemia Covid-19, ningún aspecto de la vida regresará al estado en que se encontraban previo al confinamiento, y los sistemas educativos -mayormente en América Latina y el Caribe- no serán la excepción, dado que los nuevos esquemas de aprendizaje incluirán otros saberes no solo en los educandos, sino en la comunidad educativa en general: la destreza en el 
manejo de las tecnologías, la educación socio-emocional -que ya empezó a proyectarse en algunas naciones, como nuevas asignaturas-, y con ello la configuración de las ideologías pedagógicas que permitan responder a situaciones particulares de inclusión educativa.

\section{Conclusiones}

Luego de reflexionar sobre el contexto actual educativo, y observar cómo las nuevas y diferentes modalidades virtuales para acercar los saberes trastocan otras esferas de la vida, encontramos que la educación a distancia deja al descubierto una enorme brecha digital, pues si la escuela y el aula humanizan al hombre, lo educan, lo insertan a las prácticas de socialización para tender y construir lazos entre sus pares, pareciera entonces que la educación a distancia se vislumbra actualmente como un factor deshumanizante en esta nueva modalidad, que tiende a normalizarse.

La educación crea civilización, forma conciencias, dota de saberes al hombre y lo lleva a construir y reconstruir su realidad, aun antes de la llegada de las tecnologías, más si las condiciones actuales han llevado a la sociedad a reconfigurar ese proceso de enseñanzaaprendizaje, entonces uno de los retos principales del sistema educativo es humanizar en la distancia, dejando de lado la idea de los roles esquematizados que las nuevas formas de comunicación entre docentes y estudiantes han originado con estas nuevas modalidades de conocer, compartir, aprender y evaluar.

Es claro que los nuevos aprendizajes distan mucho de obtener los mismos resultados que deja una modalidad presencial con el espacio propicio para la generación del debate y la reflexión, que motiva ese campo educador que es el aula, sin embargo el contexto actual obliga a los sistemas educativos y a los actores que en él intervienen a adaptar los diferentes estilos de enseñanza, con una visión empática, esto es, considerando cada una de las necesidades de los educandos, tomando en consideración que hoy por hoy los aprendizajes fluyen de manera individual, pues no todos los estudiantes cuentan con las mismas aptitudes y capacidades para el dominio de las herramientas tecnológicas, con los espacios adecuados para la interacción virtual, con las condiciones socio-económicas que le remiten a sumarse en la responsabilidad de aportar monetariamente para atender las necesidades más básicas de la familia.

Es por ello, que los docentes y/o educadores requieren desarrollar la habilidad de comprender las características particulares de cada niño, adolescente, joven y padre de familia interesado en continuar en el camino del saber, pero con diversas limitantes para cumplir con ello, ya sea el dominio de las tecnologías y demás herramientas, los horarios laborales que interrumpen las sesiones educativas, la calidad de la conexión, entre otras, pues el verdadero reto del docente en esta contemporaneidad es ser facilitador y al mismo tiempo efectivo proveedor de contenidos y saberes que permitan oportunamente a los educandos la reflexión de los mismos, y en consecuencia la generación de un aprendizaje significativo, a pesar de las dificultades socio-emocionales que la situación pandémica origina.

El objetivo de este análisis y propuesta de humanización de los agentes educadores, del sistema y la sociedad en general, no es sin duda alguna el criminalizar la incursión de las tecnologías en lo educativo, sino evitar los idearios de que la educación solo puede ser y presentarse en la existencia de las herramientas tecnológicas, circulando a través de los diferentes dispositivos y limitando el crecimiento, la creatividad y la expansión intelectual en aquellos que menos acceso tienen a los instrumentos del mundo globalizado, en cuanto a la red 
y la tecnología se refiere, pues de ser así, se entendería que el proceso educativo se configura en la manipulación de objetos tecnológicos y por tanto el sistema educativo estaría quebrantando uno de los principales pilares por los que existe y subsiste el posicionamiento de la educación: humanizar y civilizar a los hombres y mujeres del futuro.

\section{Referencias Bibliográficas}

Area, Manuel y Adell Jordi. (2009) e-Learning: Enseñar y aprender en espacios virtuales. En De Pablos, Juan. Tecnología Educativa. La formación del profesorado en la era de Internet. Aljibe. España. Extraído de: https://www.researchgate.net/publication/216393113_ELearning_ensenar_y_aprender_en_espacios_virtuales

CEPAL (Comisión Económica para América Latina y el Caribe) (2019). Panorama Social de América Latina, 2018. (LC/PUB.2019/3-P), CEPAL. Chile. Extraído de: https://www.cepal.org/es/publicaciones/44395-panorama-social-america-latina-2018

Rodríguez, Jorge y Coba, Juan Pablo. (2017) Impacto del m-learning en el proceso de aprendizaje: habilidades y conocimiento. Revista Iberoamericana para la Investigación y el Desarrollo Educativo (RIDE), 8 (15) México. (s/p) https://doi.org/10.23913/ride.v8i15.303

Díaz, Miguel Ángel (2020). Equipos directivos de educación primaria. Improvisar la alfabetización digital durante la cuarentena. En Casanova Cardiel Educación y pandemia: una visión académica. Instituto de Investigaciones sobre la Universidad y la Educación. México. Extraído de: http://www.iisue.unam.mx/nosotros/covid/educacion-y-pandemia Mancera, Carlos; Serna, Leslie y Barrios, Martha (2020). Pandemia: maestros, tecnología y desigualdad. Extraído de: $h$ ttps://educacion.nexos.com.mx/?p=2286

Martínez, Carmen Heedy. (2008) La educación a distancia: sus características y necesidad en la educación actual. Educación, 17 (33) Perú. (Pp. 7-27) Extraído de: http://revistas.pucp.edu.pe/index.php/educacion/article/view/1532

Silva, María Alejandra. (2020) La confrontación entre estadísticas oficiales y no oficiales: la punta del iceberg de un problema de concepción de salud. En Roncal, Ximena y Salazar, Robinson/Coords. América Latina después del 2020. Colección Insumisos Latinoamericanos elaleph.com. Argentina. www.doi.org/10.38202/americalatina2020

Torres, Mónica y López, Cecilia. (2015). Modalidades, sistemas y opciones educativas en México, ¿es posible un acuerdo de bases conceptuales? En Zubieta, Judith y Rama, Claudio/Coords. La educación a distancia en México: una nueva realidad universitaria. Virtual Educa. México. Extraído de: https://virtualeduca.org/documentos/observatorio/2015/la-educacion-a-distancia-enmexico.pdf

Trejo, Janeth (2020). La falta de acceso y aprovechamiento de los medios y las tecnologías: dos deudas de la educación en México. En Casanova Cardiel/Coord. Educación y pandemia. Una visión académica. UNAM Instituto de Investigaciones sobre la Universidad y la Educación. México. Extraído de: http://www.iisue.unam.mx/nosotros/covid/educacion-y-pandemia

Truco, Daniela y Palma, Amalia (2020). Infancia y adolescencia en la era digital: un informe comparativo de los estudios de Kids on line de Brasil, Chile, Costa Rica y el Uruguay. 
Documentos de proyectos (LC/TS.2020/18). CEPAL. Chile. Extraído de: https://www.cepal.org/es/publicaciones/45212-infancia-adolescencia-la-era-digital-uninforme-comparativo-estudios-kids-online

Viteri, Frank (2011). Educación y Tecnología: Visión filosófica de la tecnología hasta llegar a su humanización por medio de la educación. Sophia, Colección de Filosofía de la Educación, (11), Ecuador. (Pp.175-196) Extraído de: http://dspace.ups.edu.ec/handle/123456789/9392

Zebadúa, Miguel Ángel. (2020). De ciudadanos a usuarios, algunos impactos de la pandemia. En Roncal, Ximena y Salazar, Robinson /Coords. América Latina después del 2020. Colección Insumisos Latinoamericanos elaleph.com Argentina. www.doi.org/10.38202/americalatina2020 